\title{
ACTITUDES DE ESTUDIANTES UNIVERSITARIOS HACIA LA EDUCACIÓN INCLUSIVA
}

\section{ATTITUDES OF UNIVERSITY STUDENTS TOWARDS INCLUSIVE EDUCATION}

\author{
María Piedad Rivadeneira Barreiro ${ }^{1}$ \\ Boris Isaac Hernández Velásquez ${ }^{2}$ \\ Daniela Lorena Loor Lara ${ }^{3}$ \\ Mayra Monserrate Palma Villavicencio 4
}

Universidad Técnica de Manabí, Ecuador

$1 \quad$ Profesora de Inglés de la Universidad Técnica de Manabí, Ecuador. Doctora en Educación con Mención Internacional.

https://orcid.org/0000-0002-5725-6248

http://bit.ly/2MtmaeX

mprivadeneira@utm.edu.ec

$+593979153759$

2 Abogado de la Universidad Técnica de Manabí, Ecuador. Doctor en Derecho, Gobierno y Políticas Públicas. https://orcid.org/0000-0002-1057-6877

http://bit.ly/2ZaskCu

bhernandez@utm.edu.ec

$+593983346375$

3 Profesora de Inglés de la Universidad Técnica de Manabí, Ecuador. Máster Universitario en E-learning y Redes Sociales.

https://orcid.org/0000-0001-5739-5996

http://bit.ly/2H7vBfp

dlloor@utm.edu.ec

$+593998617846$

$4 \quad$ Profesora de la Universidad Técnica de Manabí, Ecuador. Magíster en Gerencia Educativa.

https://orcid.org/0000-0002-9303-6832

http://bit.ly/2HaRzyb

mpalma@utm.edu.ec

+593986371699

\section{RESUMEN}

El propósito de esta investigación es analizar las actitudes de estudiantes universitarios hacia la educación inclusiva. Con la finalidad de alcanzar este objetivo, se aplicó un estudio cuantitativo donde participaron 59 estudiantes de primer semestre de la asignatura de comunicación humana de la Carrera de Pedagogía de los Idiomas Nacionales y Extranjeros de una universidad ecuatoriana. Los estudiantes fueron escogidos sin aleatoriedad para participar de este estudio. Se aplicó estadística descriptiva para conseguir el objetivo planteado. Los resultados revelaron que gran parte de los participantes tienen actitudes positivas ante la Educación inclusiva y que unos cuantos de ellos miran desfavorablemente la misma. Las implicaciones de estos hallazgos indican que todos quienes pertenecemos a la comunidad 
educativa necesitamos más educación respecto a este tema para mejorar como sociedad y educar a otros.

Palabras clave: Educación inclusiva, formación de actitudes, agentes educativos.

\section{ABSTRACT}

The purpose of this research is to analyze the attitudes of university students towards inclusive education. With the purpose of reaching this objective, a quantitative study was applied where 59 first-semester students participated in the subject of Human Communication in the National and Foreign Language Pedagogy Career in an Ecuadorian university. The students were chosen to participate in this study without randomness. Descriptive statistics was applied to achieve the stated objective. The results revealed that many of the participants have positive attitudes towards inclusive education and that a few of them view it unfavorably. The implications of these findings indicate that all of us who belong to the educational community need more education regarding this issue to improve as a society and educate others.

KEYWORDS: Inclusive education, attitude formation, educational agents.

\section{INTRODUCCIÓN}

La globalización ha traído cambios en la educación, cambios que han llevado a introducir nuevos términos en la educación inclusiva. Así, por ejemplo, apareció el término diversidad para reemplazar al término educación especial que era un tipo de educación que únicamente consideraba a los estudiantes con discapacidades. Al referirnos a diversidad, estamos ampliando el concepto de inclusión porque se está abarcando aspectos internos y externos que confluyen en una persona (Sánchez, 2004).

La educación inclusiva es un tema que ataña a toda la comunidad educativa. Es un proceso que nos invita a cambiar políticas de Estado en materia educativa, así como prácticas y recursos educativos. De acuerdo con Serrano, Molina y Venegas (2017), la población ecuatoriana con discapacidad bordea las 816.156 personas, donde un $18 \%$ de ellos carece de instrucción. Por otro lado, el $60 \%$ de estas personas viven en zonas urbanas y el $40 \%$ restante en zonas rurales. Estos resultados nos indican los fuertes retos que tenemos que asumir para mejorar la educación de sectores relegados históricamente.

Al respecto, Echeita Sarrionandía y Sandoval Mena (2002) mencionan la exclusión social como un fenómeno latente y creciente en países en vías de desarrollo, así como los desarrollados. Este fenómeno es marcado por amplias brechas existentes en el mercado laboral que afectan especialmente a sectores pobres. La pobreza económica afecta entonces al acceso a servicios de salud, educación, vivienda, entre otros. Por otra parte, esta exclusión social mencionada por los autores antes citados puede ahondarse al evadirlos. $Y$ es que inconscientemente y sesgados por nuestros prejuicios de identificar a estudiantes destacados en ciertas áreas, podríamos caer en el error de excluir a estudiantes con capacidades diferentes.

Por otra parte, no hay que dejar de lado la importancia de educar interculturalmente. Las migraciones han traído consigo una mezcla de culturas y esto invita a fomentar la participación igualitaria de personas de diferentes culturas dentro de los sistemas educativos de cada país. Es desde el hogar que debemos educar para que no existan estas diferencias dentro de la educación. Los profesores también debemos estar formados para atender necesidades de inclusión, tal cual lo manifiestan Ruiz, Benet y Sanahuja (2017) al indicar que los educadores debemos conocer las culturas de los inmigrantes para potenciar sus capacidades dentro del proceso enseñanza-aprendizaje. 


\section{EDUCACIÓN INCLUSIVA}

Los orígenes de la inclusión en la Educación indican que todo inició en la conferencia de 1990 de la UNESCO que se llevó a cabo en Jomtien, Tailandia. Esta primera reunión que incluyó países anglosajones abrió paso a posteriores reuniones como la conferencia de Salamanca en 1994 que también fue auspiciada por la UNESCO y donde participaron 88 países y 25 organizaciones internacionales que asumieron la inclusión como principio y política educativa (Latas, 2002).

Con la globalización aparecieron exclusiones sociales, diferencias entre sectores de mejor economía en comparación con otros no tan afortunados. Esta exclusión, así como aquellas reflejadas en minorías étnicas y lingüísticas y otras propias de grupos alejados de la educación, fue lo que llevó a reconsiderar la inclusión más allá de contextos donde las poblaciones tenían necesidades educativas especiales. Todo esto apoyado en la Declaración Mundial de los Derechos Humanos que en su artículo 26 hace referencia al derecho de toda persona a tener acceso a la educación (Canet, 2009).

Lo referido anteriormente visibiliza la necesidad de una educación democrática, educación incluyente donde se responda a problemas sociales. Las escuelas no son los únicos agentes de cambio. Es importante involucrar a padres de familia, autoridades políticas y comunidad en general. Se pretende romper paradigmas y trabajar sobre políticas educativas que brinden igualdad de derechos para acceder a espacios educativos. Reformar currículos y prácticas de enseñanza-aprendizaje son también reformas que fomentan igualdad de derechos en educación, tolerancia y empatía hacia grupos que históricamente han sido relegados en los sistemas educativos.

Actualmente, la inclusión educativa es un tema poco abordado en las universidades, tal cual lo refieren de la Herrán, Paredes Labra, y Monsalve Treskow (2017), al indicar que es manejado desde la enseñanza o la investigación por docentes que quieren enfatizar que si no hay inclusión no hay educación. Entonces nos encontramos con un problema no resuelto en las escuelas que se extiende a niveles superiores en la educación. $Y$ es que al no existir una cultura institucional donde se fomente la inclusión, no se produce cambios para mejorar la educación.

La Universidad Técnica de Manabí, lugar donde se desarrolló la presente investigación, es una universidad que ha buscado incluir a personas con discapacidad, tanto así que su porcentaje de inclusión de $12.14 \%$ la ubica por encima de la media nacional del Ecuador. Es una universidad que fomenta la inclusión en sus políticas educativas contando con 53 estudiantes y 16 profesores con diferentes discapacidades (De la Herrán, Paredes Labra, y Monsalve Treskow, 2017). De esta manera se sigue la línea de la UNESCO (2005) donde se atiende a la diversidad de estudiantes con el fin de evitar su exclusión.

\section{ACTITUDES HACIA LA EDUCACIÓN INCLUSIVA}

El éxito de las prácticas inclusivas depende mucho de sus agentes educativos. De ahí que las actitudes que ellos demuestren hacia la inclusión van a favorecer o afectar la educación. De acuerdo con Granada Azcárraga, Pomés Correa y Sanhueza Henríquez (2013), la actitud es "un conjunto de percepciones, creencias, sentimientos a favor o en contra y formas de reaccionar ante la postura educativa que centra su esfuerzo en el logro de los aprendizajes de todos los estudiantes" (p. 53). De ahí que las actitudes positivas de los agentes educativos juegan un papel importante en la implantación de prácticas inclusivas exitosas.

La actitud del docente hacia la inclusión es una variable que puede verse afectada por otras 
tales como el tipo de discapacidad, edad, sexo, formación, años de experiencia en prácticas de inclusión, nivel educativo en el que se trabaja y los recursos disponibles en la educación (Tárraga Mínguez, Grau Rubio y Peirats Chacón, 2013). Adicionalmente, hay que considerar los componentes cognitivo, afectivo y conductual. El primer componente destaca las creencias sobre un objeto específico, el segundo componente implica las emociones que están ligadas a esas creencias y el tercer componente menciona la conducta que refleja la actitud (Clavijo, López, Cedillo, Mora y Ortiz, 2016).

Por el lado de los estudiantes, hay evidencia de estudios como el de Suriá Martínez (2011) que resalta la actitud positiva de estudiantes universitarios hacia sus compañeros con discapacidad. Además, indica la importancia de la interacción con estudiantes discapacitados para sensibilizar a los demás compañeros (como lo cita Novo-Corti, Muñoz-Cantero y CalvoPorral, 2011). Pero además de las actitudes hacia la discapacidad, existen las actitudes hacia diferentes culturas o grupos minoritarios como el de LGBTI. Este es un aspecto en el cual debemos ser educados desde pequeños para tener actitud positiva a grupos minoritarios que han sido excluidos a lo largo de la historia.

\section{METODOLOGÍA}

\section{OBJETIVOS Y PREGUNTAS DE INVESTIGACIÓN}

Las preguntas de investigación planteadas en este estudio fueron las siguientes:

PI 1. ¿Cuáles son las actitudes de estudiantes universitarios hacia la educación inclusiva?

PI 2. ¿Qué prácticas inclusivas se podrían implantar a partir de las actitudes positivas de los estudiantes universitarios hacia la educación inclusiva?

En base a estas preguntas de investigación, se estableció el objetivo de analizar las actitudes de estudiantes universitarios hacia la educación inclusiva. Para alcanzar este objetivo, se plantearon los siguientes objetivos específicos:

Objetivo 1: Identificar las actitudes de estudiantes universitarios hacia la educación inclusiva.

Objetivo 2: Analizar las actitudes positivas de los estudiantes universitarios hacia la educación inclusiva para incentivar prácticas de inclusión.

\section{PARTICIPANTES}

Los participantes de esta investigación fueron estudiantes de primer semestre de la asignatura de comunicación humana de la Carrera de Pedagogía de los Idiomas Nacionales y Extranjeros de una universidad ecuatoriana. Cincuenta y nueve estudiantes, cuyas edades oscilaban entre 18 y 42 años, fueron escogidos sin aleatoriedad para participar de este estudio. Los grupos pertenecían a dos cursos ya formados de la Carrera antes mencionada. Los estudiantes firmaron fichas de consentimiento donde indicaban su participación consciente y voluntaria en este estudio.

\section{INSTRUMENTOS}

Para llevar a cabo esta investigación, se entregó a los participantes un cuestionario en papel de Vélez Latorre (2013) con 33 declaraciones relacionadas a actitudes hacia la educación inclusiva. El cuestionario contenía una escala de Likert que iba desde totalmente de acuerdo a totalmente en desacuerdo con las declaraciones, en donde los estudiantes tenían que escoger la opción que reflejaba su actitud con respecto a la educación inclusiva.

\section{PROCEDIMIENTO DE RECOLECCIÓN DE DATOS}

Una vez los estudiantes contestaron las preguntas del cuestionario, se procedió a la recolección de datos. Los datos fueron 
tabulados para identificar las actitudes de estudiantes universitarios hacia la educación inclusiva y analizar las actitudes positivas hacia la educación inclusiva y de esa manera incentivar prácticas de inclusión.

\section{ANÁLISIS DE DATOS}

Los datos fueron analizados manualmente detectando las respuestas con más impacto en cada una de las declaraciones.

\section{RESULTADOS}

Los resultados de esta investigación revelaron que gran parte de los participantes tienen actitudes positivas hacia la educación inclusiva, respondiendo así a la pregunta de investigación 1. Así por ejemplo 51 participantes de 59 estuvieron totalmente de acuerdo en que el docente de un aula inclusiva requiere conocer estrategias pedagógicas diferentes. Luego 47 estudiantes de 59 indicaron que la educación inclusiva favorece el aprendizaje, la participación y la convivencia de todos los estudiantes del aula.

Por otro lado, 31 estudiantes de 59 señalaron estar totalmente de acuerdo con que en las aulas inclusivas se trabajan contenidos más variados y llamativos. Cuarenta y tres participantes de 59 estuvieron totalmente de acuerdo en que todas las escuelas deberían ser inclusivas. Relacionada a la afirmación anterior, 23 participantes estuvieron totalmente de acuerdo en cualificarse para asumir un aula inclusiva, 18 participantes indicaron estar medianamente de acuerdo, 14 ser indiferentes, 2 medianamente en desacuerdo y 2 en total desacuerdo.

En lo referente a que los estudiantes tengan acceso a educación especializada de acuerdo a sus características, 41 participantes de 59 indicaron estar totalmente de acuerdo en que así tendrían mejores procesos de aprendizaje y participación. También mostraron actitud positiva y disposición a participar de procesos de inclusión cuando 33 estudiantes de 59 estuvieron totalmente de acuerdo en su disposición a emplear organizaciones flexibles de aula para favorecer el aprendizaje y participación de todos sus estudiantes.

Así mismo, la actitud positiva hacia la inclusión se evidenció cuando 44 participantes de 59 indicaron estar totalmente de acuerdo en acondicionar su aula de clase para facilitar el aprendizaje y participación de todos sus estudiantes. Por otro lado, 42 estudiantes manifestaron estar totalmente de acuerdo en que las escuelas deben implementar estrategias de educación continua para asumir la educación inclusiva. En lo referente a si las escuelas deberían tener aulas especializadas de acuerdo con las particularidades de los estudiantes, 39 estudiantes de 59 estuvieron totalmente de acuerdo con esta afirmación.

En la afirmación sobre si los agrupamientos de los estudiantes de formas variadas favorecen la atención a la diversidad en el aula inclusiva, 28 estudiantes estuvieron totalmente de acuerdo y otros 17 participantes manifestaron estar de acuerdo. En lo referente a su convencimiento de que el empleo de estrategias inclusivas mejora el clima del aula, 33 participantes estuvieron totalmente de acuerdo con esta afirmación. Un número considerable de participantes también demostraron su sensibilidad ante este tema cuando 27 de los participantes estuvieron totalmente de acuerdo en que las escuelas no cuentan con suficiente material para atender las particularidades de un aula inclusiva.

También, 25 de los participantes indicaron estar totalmente de acuerdo en que se sentirían frustrados si la escuela no les suministrara los recursos requeridos para atender un aula inclusiva, mientras que 18 de los participaron sólo indicaron estar de acuerdo con esta afirmación. Por otro lado, 19 participantes de 59 estuvieron totalmente de acuerdo en que sin material especializado no pueden desarrollar 
adecuadamente los procesos de enseñanza en un aula inclusiva, mientras que otros 18 participantes indicaron sólo estar de acuerdo con esta afirmación. Finalmente, 41 participantes de 59 estuvieron totalmente de acuerdo en estar dispuestos a trabajar por proyectos de aula, rincones de aprendizaje u otra organización flexible que favorezca el aprendizaje y participación de todos los estudiantes.

Contrario a estas actitudes positivas, hubo participantes que mostraron actitudes poco favorables a la inclusión. Por citar, 23 participantes de 59 indicaron estar totalmente de acuerdo en que les preocupa que un aula inclusiva no alcance a dar todos los contenidos del plan de estudio. Veintiún participantes estuvieron de acuerdo con esta afirmación. En lo que respecta a que les molesta que las escuelas no puedan determinar de forma autónoma qué estudiantes ingresan a esta, 18 participantes estuvieron totalmente de acuerdo con la afirmación, mientras 15 estuvieron de acuerdo. Finalmente, demostraron poco interés por implementar cambios en las estrategias de enseñanza cuando 18 participantes indicaron estar totalmente de acuerdo en que como docentes de aulas inclusivas seguirían empleando las mismas estrategias de enseñanza que conocen, mientras 15 participantes señalaron estar de acuerdo.

Con estos resultados, se respondió a la pregunta de investigación 2, la misma que busca implantar prácticas inclusivas a partir de las actitudes positivas de los estudiantes universitarios hacia la educación inclusiva. Investigaciones desarrolladas por De la Herrán, Pinargote y Véliz (2016), García y Álava (2018), entre otros, ayudaron a explorar formas de alcanzar el objetivo asociado a dicha pregunta de investigación. Por otro lado, aplicando las dimensiones del Index sugeridas por Booth y Ainscow (2002), se pretende trabajar colaborativamente dentro de la comunidad universitaria y fuera de esta para lograr metas educativas que consideren prácticas inclusivas.

Desde esta perspectiva, se siguen las etapas del Index: Definir un equipo de plan de mejora con un coordinador que informe al resto de profesorado sobre el Index, materiales y metodología. Esto se realiza con la finalidad de informar y recoger opiniones que favorezcan el proceso de inclusión. Posteriormente se usan los materiales para analizar las prioridades de la institución educativa. En la tercera etapa se busca implantar un plan de mejoras para en la etapa 4 apoyar las innovaciones. Este proceso es finalmente evaluado tomando en consideración el desarrollo de culturas, políticas y prácticas inclusivas.

Como parte del plan de mejoras se consideran las dimensiones del Index: Crear culturas inclusivas (construir comunidad y establecer valores inclusivos), elaborar políticas inclusivas (desarrollar una escuela para todos y organizar la atención a la diversidad), y desarrollar prácticas inclusivas (dirigir el proceso de aprendizaje y movilizar recursos). Es importante indicar que el Index debe ser adaptado a nuestro contexto y necesidades, por lo que requiere que el equipo de plan de mejoras analice cuidadosamente los materiales y la metodología a usarse.

\section{DISCUSIÓN Y CONCLUSIONES}

Con los resultados obtenidos, se puede indicar que, aunque la muestra no es representativa para generalizar los hallazgos dentro de la comunidad científica, es evidente que hay cambios en la educación que están rompiendo viejos paradigmas. Sin duda alguna, las nuevas generaciones están aceptando la educación inclusiva como parte de los procesos de enseñanza aprendizaje donde reconocemos nuestras diferencias y potenciamos las habilidades de cada persona.

Son ya tres décadas desde que el término 
inclusión se consideró en la Educación en la primera conferencia de la UNESCO que se llevó a cabo en Jomtien, Tailandia. Los trabajos de la comunidad educativa están dando frutos, los mismos que se avistan con la aceptación de grupos minoritarios que han sido históricamente relegados de los procesos educativos. El nivel educativo de los padres y el trabajo coordinado de autoridades educativas, de gobierno y la comunidad ha permitido superar gran parte de las diferencias educativas que especialmente ocurren en países que se encuentran en vías de desarrollo.

Es importante manifestar que la educación inclusiva supone un enriquecimiento ideológico y conceptual donde se pretende corregir errores asociados a la integración del alumnado, tal cual lo manifiesta Escribano y Martínez (2013). De ahí que este estudio pretende partir de una visión de los que directamente interactúan con grupos que han sido excluidos y así comprender lo que necesitamos mejorar como sociedad. Es un llamado a nosotros como docentes e investigadores a continuar la labor que empezó tres décadas atrás de manera que se potencien las habilidades de cada estudiante, se fomente el trabajo cooperativo y se resalte la parte humana de nuestra sociedad.

\section{REFERENCIAS BILOGRÁFICAS}

Booth, T., \& Ainscow, M. (2002). Guía para la evaluación y mejora de la educación inclusiva. Consorcio Universitario para la Educación Inclusiva. Universidad Autónoma de Madrid, 16.

Canet, G. V. (2009). Construyendo un concepto de educación inclusiva: una experiencia compartida. Aspectos clave de la Educación Inclusiva, 13.

Clavijo, R., López, C., Cedillo, C., Mora, C., \& Ortiz, W. (2016). Actitudes docentes hacia la educación inclusiva en Cuenca. Maskana, 7(1), 13-22.
De la Herrán, A., Paredes Labra, J., y Monsalve Treskow, D. V. (2017). Cuestionario para la evaluación de la educación inclusiva universitaria (CEEIU). Revista Complutense de Educación.

De la Herrán Gascón, A., Ortega, M. P., \& Briones, V. V. (2016). Génesis de una universidad inclusiva en Ecuador. La Universidad Técnica de Manabí. Revista Iberoamericana de Educación, 70(2).

Echeita Sarrionandía, G., \& Sandoval Mena, M. (2002). Educación inclusiva o educación sin exclusiones. Revista de educación, 327, 31-48.

Escribano, A., \& Martínez, A. (2013). Inclusión educativa y profesorado inclusivo: aprender juntos para aprender a vivir juntos, 122. Madrid: Narcea Ediciones.

García, E., \& Álava, L. (2018). Educación inclusiva y calidad de vida de los estudiantes con discapacidad motriz de la universidad técnica de Manabí. Revista Atlante.

Granada Azcárraga, M., Pomés Correa, M. P., \& Sanhueza Henríquez, S. (2013). Actitud de los profesores hacia la inclusión educativa. p. 53.

Latas, Á. P. (2002). Acerca del origen y sentido de la educación inclusiva. Revista de educación, 327(1), 11-29.

Novo-Corti, I., Muñoz-Cantero, J. M., \& CalvoPorral, C. (2011). Análisis de las actitudes de los jóvenes universitarios hacia la discapacidad: un enfoque desde la teoría de la acción razonada. Relieve-Revista Electrónica de Investigación y Evaluación Educativa, 17(2).

Ruiz, M., Benet, A., y Sanahuja, A. (2017). Percepción y Formación sobre Educación Intercultural e Inclusiva de los Estudiantes de Magisterio de Castellón y Tucumán. En P. García-Sempere, M. Pinargote, V. Véliz, A. de la Herrán, y B. Montiano (coords.), Formación 
y transformación para la educación inclusiva en la universidad (pp. 27-52). Granada: Editorial Universidad de Granada.

Sánchez, A. (2004). La educación inclusiva: dilemas y desafíos. Educación, desarrollo y diversidad, 7(2), 25-40.

Serrano, B., Molina, J., y Venegas, G. (2017). Percepción y Formación sobre Educación Intercultural e Inclusiva de los Estudiantes de Magisterio de Castellón y Tucumán. En P. García-Sempere, M. Pinargote, V. Véliz, A. de la Herrán, y B. Montiano (coords.), Formación y transformación para la educación inclusiva en la universidad (pp. 27-52). Granada: Editorial Universidad de Granada.
Tárraga Mínguez, R., Grau Rubio, C., y Peirats Chacón, J. (2013). Actitudes de los estudiantes del Grado de Magisterio y del Máster de Educación Especial hacia la inclusión educativa. Revista electrónica interuniversitaria de formación del profesorado, 2013, vol. 44, num. 16, p. 55-72.

UNESCO (2005) Guidelines for inclusion. Ensuring access to education for all. París: UNESCO.

Vélez Latorre, L. (2013). La educación inclusiva en docentes en formación: su evaluación a partir de la teoría de facetas. Folios, (37).

\section{APÉNDICE}

\section{ACTITUDES DE ESTUDIANTES UNIVERSITARIOS HACIA LA EDUCACIÓN INCLUSIVA}

El presente instrumento tiene como propósito identificar sus actitudes sobre la educación inclusiva. Se entiende la educación inclusiva como la posibilidad de acoger en la institución educativa a todos los estudiantes valorando la diversidad de sus características personales y culturales.

Por favor complete los siguientes datos de identificación:

Género: Edad:

Carrera: Semestre académico que cursa:

A continuación, encontrará una serie de afirmaciones las cuales debe leer cuidadosamente. Marque con una X la casilla correspondiente al nivel de acuerdo o desacuerdo con las afirmaciones enunciadas sobre la educación inclusiva, teniendo en cuenta las siguientes opciones:

- Totalmente de acuerdo (TA)

- Medianamente de acuerdo (MA)

- Indiferente (I)

- Medianamente en desacuerdo (MD)

- Totalmente en desacuerdo (TD) 
1. El docente de un aula inclusiva requiere conocer estrategias pedagógicas diferentes.

TA MA I MD TD

2. Creo que la educación inclusiva está dirigida únicamente a los estudiantes con discapacidad y aquellos pertenecientes a minorías étnicas.

TA MA I MD TD

3. El éxito de la educación inclusiva depende exclusivamente del docente.

TA MA I MD TD

4. La educación inclusiva favorece el aprendizaje, la participación y la convivencia de todos los estudiantes del aula.

TA MA I MD TD

5. Los únicos estudiantes beneficiados en un aula inclusiva son aquellos con discapacidad y/o dificultades de aprendizaje.

TA MA I MD TD

6. Es más difícil el manejo de la disciplina en un aula inclusiva que en un aula regular/ tradicional.

TA MA I MD TD

7. En las aulas inclusivas se trabajan contenidos más variados y llamativos.

TA MA I MD TD

8. Todas las escuelas deberían ser inclusivas.

TA MA I MD TD

9. Desearía poder trabajar únicamente en un aula regular/tradicional.

TA MA I MD TD

10. Me preocupa que en un aula inclusiva deba invertir mucho tiempo en los estudiantes que tienen mayores dificultades.

TA MA I MD TD

11. Me preocupa que en un aula inclusiva no alcance a dar todos los contenidos del plan de estudio.

TA MA I MD TD

12. Me inquieta que la educación inclusiva sea una política educativa obligatoria.

TA MA I MD TD 
13. Me molesta que las escuelas no puedan determinar de forma autónoma qué estudiantes ingresan a esta.

TA MA I MD TD

14. Me preocupa que todas las aulas se transformen en inclusivas.

TA MA I MD TD

15. Estoy dispuesto a cualificarme para asumir un aula inclusiva.

TA MA I MD TD

16. Si los estudiantes pudieran tener acceso a educación especializada de acuerdo a sus características, tendrían mejores procesos de aprendizaje y participación.

TA MA I MD TD

17. Yo no sugeriría una escuela inclusiva para un estudiante normal.

TA MA I MD TD

18. Estoy dispuesto a emplear organizaciones flexibles de aula para favorecer el aprendizaje y participación de todos mis estudiantes.

TA MA I MD TD

19. Acondicionaría mi aula de clase para facilitar el aprendizaje y participación de todos los estudiantes.

TA MA I MD TD

20. Las escuelas deben implementar estrategias de educación continua para asumir la educación inclusiva.

TA MA I MD TD

21. Las escuelas deberían tener aulas especializadas de acuerdo con las particularidades de los estudiantes.

TA MA I MD TD

22. Debería haber escuelas inclusivas y escuelas regulares/tradicionales.

TA MA I MD TD

23. En las aulas inclusivas se deben emplear las mismas estrategias didácticas que en un aula regular/ tradicional.

TA MA I MD TD 
24. Siento que los ambientes de aprendizaje no son esenciales para el desarrollo exitoso de las aulas inclusivas.

TA MA I MD TD

25. Los agrupamientos de los estudiantes de formas variadas favorecen la atención a la diversidad en el aula inclusiva.

TA MA I MD TD

26. Me preocupa que las estrategias necesarias para un aula inclusiva sean muy complejas y dispendiosas para desarrollar.

TA MA I MD TD

27. Estoy convencido que el empleo de estrategias inclusivas mejora el clima del aula.

TA MA I MD TD

28. Las escuelas no cuentan con el suficiente material para atender las particularidades de un aula inclusiva.

TA MA I MD TD

29. Me sentiría frustrado si la escuela no me suministrara los recursos requeridos para atender un aula inclusiva.

TA MA I MD TD

30. Me molesta tener que utilizar diferentes formas de evaluar los procesos de aprendizaje de mis estudiantes.

TA MA I MD TD

31. Como docente de aula inclusiva seguiría empleando las mismas estrategias de enseñanza que conozco.

\section{TA MA I MD TD}

32. Sin material especializado no puedo desarrollar adecuadamente los procesos de enseñanza en un aula inclusiva.

\section{TA MA I MD TD}

33. Estaría dispuesto a trabajar por proyectos de aula, rincones de aprendizaje u otra organización flexible que favorezca el aprendizaje y participación de todos los estudiantes.

TA MA I MD TD

Gracias por su colaboración. 